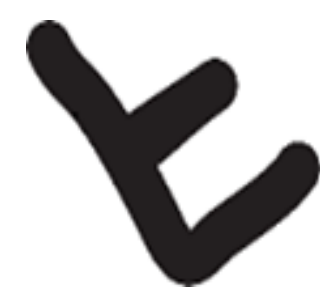

REVISTA DE ESTUDIOS E INVESTIGACIÓN EN PSICOLOGÍA Y EDUCACIÓN

ISSN: 1138-1663; eISSN: 2386-7418

2020, Vol. 7, No. 2, 127-135.

DOI: https://doi.org/10.17979/reipe.2020.7.2.6985

\title{
Viveza, control de imagen, y actitudes ante la muerte en adolescentes y adultos
}

\author{
Image vividness, image control and attitudes towards death \\ among teenagers and adults
}

\author{
María Esther López-Pérez*, Margarita Pino-Juste (D)*, Alfredo Campos (D)** \\ *Universidad de Vigo, **Universidad de Santiago de Compostela
}

\begin{abstract}
Resumen
Las personas pueden tener imágenes mentales vivas sobre la muerte, y pueden tener control sobre ellas. El objetivo de esta investigación era saber si la viveza de imagen y el control de imagen influían en la actitud ante la muerte en un grupo de adolescentes (Estudio 1) y en un grupo de adultos (Estudio 2). Las variables independientes fueron la viveza de imagen y el control de imagen de los participantes, y como variables dependientes se utilizaron las subescalas del Death Attitude Profile-Revised (DAP-R): Aceptación de Acercamiento (AA), Miedo a la Muerte (FD), Evitación de la Muerte (DA), Aceptación de Escape (EA), y Aceptación Neutral (NA). Se encontró que, en el grupo de adolescentes, los individuos que tenían un mayor control de imagen tuvieron significativamente menos Miedo a la Muerte (FD), menores puntuaciones en la escala de Evitación de la Muerte (DA), y mayores puntuaciones en la escala de Aceptación Neutral (NA) que los estudiantes que tuvieron un menor control de imagen. En el Estudio 2 no se encontró influencia de la viveza de imagen ni del control de imagen sobre las subescalas del Death Attitude Profile-Revised (DAP$\mathrm{R})$. Se analizan las repercusiones de estos resultados en el ámbito escolar.
\end{abstract}

Palabras clave: imagen mental; actitud ante la muerte; educación sobre la muerte

Abstract

People can have vivid mental images of death and they can have control over them. The aim of this study was to examine the influence of image vividness and image control on attitudes towards death among a group of teenagers (Study 1) and a group of adults (Study 2). The independent variables were image vividness and image control, and the subscales of the Death Attitude Profile-Revised (DAP-R) questionnaire were used as dependent variables: Acceptance of Approach (AA), Fear of Death (FD), Death Avoidance (DA), Escape Acceptance (EA), and Neutral Acceptance (NA). The results showed that teenage subjects with greater image control displayed significantly less Fear of Death (FD) and obtained lower scores in Avoidance of Death (DA) and higher scores in Neutral Acceptance (NA) than students with lower image control. In Study 2, image vividness and image control were not found to influence the subscales of the Death Attitude Profile-Revised (DAP-R). The article also analyses the implications of the findings in an educational context.

Keywords: imagery; death attitudes; death education

María Esther López-Pérez y Margarita Pino-Juste (D): Departamento de Didáctica y Organización Escolar y Métodos de Investigación,

Facultad de Educación, Universidad de Vigo. Campus As Lagoas - 32004 Ourense, España.

Alfredo Campos (D): Departamento de Psicología Básica, Facultad de Psicología, Universidad de Santiago de Compostela. Campus

Vida - 15782 Santiago de Compostela, España

Correspondencia relativa a este artículo: Alfredo Campos - alfredo.campos@usc.es 
Las imágenes mentales son experiencias semejantes a la percepción, que se producen cuando están ausentes todos o parte de los estímulos que están representados (Campos, 1998). Las personas pueden tener imágenes muy vivas (cuando son tan vivas como la misma realidad), o pueden ser muy vagas. Asimismo, pueden tener un control de las imágenes (cuando una imagen la podemos tener tiempo en nuestra mente y "verla" desde distintas perspectivas o posiciones), o pueden ser imágenes sobre las que no tenemos un dominio, sino que vienen y se van (Campos y Canto, 2019).

Las imágenes mentales juegan un papel importante en todos los procesos cognitivos, y como consecuencia, en los comportamientos (Campos y Campos-Juanatey, 2019; Campos y González, 2017; Pérez-Fabello y Campos, 2017; Pérez-Fabello et al., 2018). Las imágenes mentales, entendidas no sólo como imágenes visuales, sino también, como auditivas, gustativas, olfativas, táctiles, etc., afectan directamente a las actitudes (Campos y Fuentes, 2016; Campos y Pérez-Fabello, 2005), y tienen un efecto superior sobre ellas al que tiene el lenguaje (Holmes y Mathews, 2005).

Las actitudes hacia la muerte están muy relacionadas con muchos factores, como puede ser la edad del individuo, la cultura, la raza, la educación, el género, y otras variables que van modificando la relación entre el individuo y la muerte (Lee, 2009; McGovern y Barry, 2000). Una variable que influye mucho en la actitud del individuo hacia la muerte son las imágenes mentales, sobre todo si la imagen mental que tiene el individuo sobre la muerte es positiva o negativa (Borkovec et al., 2004).

En la revisión bibliográfica no hemos encontrado ninguna investigación en la que se relacione la viveza de imagen o el control de imágenes con las actitudes ante la muerte; sin embargo, hay mucha bibliografía en la relación entre las imágenes mentales y la ansiedad ante la muerte, miedo a la muerte y actitud hacia la muerte (Berna et al., 2011; Holmes et al., 2008; Nilsson et al., 2012). Tanto la capacidad que tiene el individuo de formar imágenes mentales muy vivas, como la capacidad de controlarlas, influye en muchos comportamientos del individuo y en muchos procesos cognitivos (Berna et al., 2011; Deeprose y Holmes, 2010; Hales et al., 2011; Holmes et al., 2008; Pérez-Fabello y Campos, 2017; Pérez-Fabello et al., 2018), y en muchos procesos terapéuticos (Borkovec et al., 2004; Clark et al., 2006; Dönmez y Bilgin, 2020).

Las personas pueden tener una imagen positiva o negativa hacia la muerte, esa imagen va a hacer que la persona tenga una actitud positiva o negativa hacia la muerte. En muchos casos, cuando la imagen de la muerte es muy negativa y la actitud ante la muerte también es muy negativa, se pueden producir en el individuo desórdenes mentales (Brewin et al., 2010). En estas circunstancias, muchos individuos se niegan a utilizar las imágenes mentales porque producen mucha ansiedad (WeBlau et al., 2015); sin embargo, es necesario que la persona controle esas imágenes y, mediante técnicas terapéuticas con utilización de imágenes mentales, cambie la imagen negativa de la muerte por una imagen positiva (Stopa et al., 2012). Al cambiar la imagen, de negativa a positiva, las personas también cambian su actitud ante la muerte (Ng et al, 2014; Nilsson et al, 2012; Stopa et al., 2012; Wild et al., 2008).

Dönmez y Bilgin (2020) efectuaron una investigación en la que se pretendía evaluar el efecto de la imagen guiada sobre la ansiedad, la empatía y la actitud de las enfermeras hacia pacientes oncológicos terminales. Los autores encontraron que el grupo al que se le aplicó la imagen guiada obtuvo un mayor incremento de las actitudes positivas hacia los pacientes oncológicos terminales, superior a las puntuaciones que obtuvo el grupo control. Este grupo vio dos películas sobre las emociones y pensamientos de los enfermos terminales de cáncer.

Recientemente, López-Pérez et al. (2020a) efectuaron una investigación en la que deseaban averiguar si la imagen de la muerte (positiva o negativa) que tienen las personas influía en la ansiedad ante la muerte y en distintas subescalas del Death Attitude Profile-Revised (DAP-R; Wong et al., 1994). Encontraron, en un grupo de adolescentes, que los individuos con una imagen positiva de la muerte tenían menos ansiedad ante la muerte que las personas con una imagen negativa. Los estudiantes que tenían una imagen positiva de la muerte tenían una mayor Aceptación de Acercamiento (AA), menor Miedo a la Muerte (FD), menor Evitación de la Muerte (DA), mayor Aceptación de Escape (EA), y mayor Aceptación Neutral (NA) que los que tenían una imagen negativa de la muerte.

En otra investigación, López-Pérez et al. (2020b) deseaban averiguar si la imagen de la muerte (positiva o negativa) influía significativamente en la actitud hacia la muerte en un grupo de estudiantes universitarios. La 
actitud hacia la muerte la midieron mediante las subescalas del Death Attitude Profile-Revised (DAP-R; Wong et al., 1994). Encontraron que los estudiantes con una imagen positiva de la muerte tenían menos Miedo a la Muerte (FD), menos Evitación de la Muerte (DA), más Aceptación de Escape (EA), y mayor puntuación en la Aceptación Neutral (NA) que los individuos que tuvieron una imagen negativa de la muerte.

\section{Estudio 1}

El objetivo de esta investigación era saber si la viveza de imagen y el control de imagen influían en la actitud de un grupo de adolescentes ante la muerte. Se pensaba que la viveza de imagen influiría incrementando las emociones y las actitudes, y que el control de imagen influiría controlando las emociones negativas y, por lo tanto, también las actitudes. Para ello, formulamos la hipótesis de que, tanto la imagen como el control de imagen que tienen los participantes, influyen significativamente en la actitud ante la muerte. Las variables independientes son la viveza de imagen y el control de imagen de los participantes, y como variables dependientes se utilizaron las subescalas del Death Attitude Profile-Revised (DAP-R): Aceptación de Acercamiento (AA), Miedo a la Muerte (FD), Evitación de la Muerte (DA), Aceptación de Escape (EA), y Aceptación Neutral (NA).

\section{Método}

\section{Participantes}

Un grupo de 280 estudiantes de Educación Secundaria Obligatoria (130 mujeres y 150 hombres), que estudiaban en colegios públicos y concertados, participaron en esta investigación. Los participantes tenían una media de edad de 14.90 años, una desviación típica de 2.60, y un rango de 12 a 17 años.

\section{Instrumentos}

Vividness of Visual Imagery Questionnaire (Marks, 1973), versión española (Campos et al., 2002): Consta de 16 ítems que presentan distintas situaciones, y se pide a los participantes que puntúen la viveza de imagen en una escala de 5 puntos, que oscila entre 5 = "ninguna imagen, tú sólo conoces lo que estás pensando del objeto", y 1 = "imagen perfectamente clara y tan viva como una visión normal". Una puntuación baja indica alta viveza de imagen y viceversa. Los participantes deben cubrir el test dos veces, la primera con los ojos abiertos, y la segunda con ellos cerrados. Este test no tiene límite de tiempo. En el estudio realizado por Campos et al. (2002) se obtuvo un coeficiente alfa de Cronbach de .88.

Gordon Test of Visual Imagery Control (TVIC; Richardson, 1969), versión española (Pérez-Fabello y Campos, 2004): Consta de 12 ítems a través de los cuales se pide a los participantes que representen mentalmente un coche en distintas posiciones. La respuesta "si" se puntúa con dos puntos, la respuesta "inseguro" se puntúa con un punto, y la respuesta "no", se puntúa con cero puntos. El test mide la capacidad de control de imagen que tienen los participantes. La puntuación puede oscilar entre 0 y 24 puntos.

Death Attitude Profile-Revised (DAP-R; Wong et al., 1994): Es una versión del Death Attitude Profile (DAP, Gesser et al., 1988). Consta de 32 ítems que se puntúan en una escala tipo Likert, que va de $1=$ "fuertemente desagradable" a 7 = "fuertemente agradable". Las subescalas son: Miedo a la Muerte (Fear of Death, FD) (Por ejemplo, "Tengo un miedo intenso a la muerte"), Evitación de la Muerte (Death Avoidance, DA) (Por ejemplo, "Evito totalmente pensar en la muerte"), Aceptación Neutral (Neutral Acceptance, NA) (Por ejemplo, "La muerte es un aspecto natural de la vida"), Aceptación de Acercamiento (Approach Acceptance, AA) (Por ejemplo, "Espero una vida después de la muerte"), y Aceptación de Escape (Escape Acceptance, EA) (Por ejemplo, "La muerte proporciona un escape de este mundo terrible"). El test no tiene límite de tiempo.

\section{Procedimiento}

A los participantes, en sus clases habituales, se les aplicó la versión española (Pérez-Fabello y Campos, 2004) del Gordon Test of Visual Imagery Control (TVIC; Richardson, 1969), la versión española (Campos et al., 2002) del Vividness of Visual Imagery Questionnaire (Marks, 1973), y la versión española del Death 
Attitude Profile-Revised (DAP-R; Wong et al., 1994). El orden de presentación de los tests se contrabalanceó.

Todos los estudiantes participaron de manera voluntaria en el estudio, no tuvieron que escribir ningún dato identificativo y se les garantizó la confidencialidad de los resultados. Las pruebas se pasaron en el horario habitual de clase, en grupos de, aproximadamente, 20 alumnos. El estudio se realizó de acuerdo con las normas éticas contenidas en la Declaración de Helsinki de 2013, y se ha recibido la aprobación del Comité Ético de la Universidad de Santiago de Compostela.

Hemos clasificado a los estudiantes en altos y bajos en control de imagen cuando sus puntuaciones en el Gordon Test of Visual Imagery Control estaban por encima o por debajo, respectivamente, de la media del grupo. Y, hemos clasificado a los estudiantes en altos y bajos en viveza de imagen cuando sus puntuaciones en el Vividness of Visual Imagery Questionnaire (Marks, 1973) estaban por encima o por debajo de la media del grupo.

\section{Análisis de Datos}

Los análisis estadísticos se llevaron a cabo con el programa IBM SPSS 22.0. En primer lugar, se obtuvo el alfa de Cronbach para conocer la fiabilidad del Vividness of Visual Imagery Questionnaire (Marks, 1973), del Gordon Test of Visual Imagery Control (TVIC; Richardson, 1969), y de las subescalas del Death Attitude Profile-Revised (DAP-R; Wong et al., 1994). Por otro lado, para poder comprobar si la viveza de imagen y el control de imagen influían en cada una de las subescalas del Death Attitude Profile-Revised (DAP-R), se realizó un Análisis Multivariado de Varianza (MANOVA) de 2 (Viveza de imagen) x 2 (Control de imagen) facotres. Se utilizaron como variables dependientes las siguientes subescalas del Death Attitude ProfileRevised (DAP-R): Aceptación de Acercamiento (AA), Miedo a la Muerte (FD), Evitación de la Muerte (DA), Aceptación de Escape (EA), y Aceptación Neutral (NA).

\section{Resultados}

La fiabilidad del Vividness of Visual Imagery Questionnaire (VVIQ), medida a través del alfa de Cronbach fue de .91, y la del Gordon Test of Visual Imagery Control (TVIC; Richardson, 1969) fue .68. La fiabilidad de las distintas subescalas del Death Attitude Profile-Revised (DAP-R) fueron las siguientes: Aceptación de Acercamiento (AA): $\alpha=.87$; Miedo a la Muerte (FD) : $\alpha=.86$; Evitación de la Muerte (DA) : $\alpha=.86$; Aceptación de Escape (EA) : $\alpha=.78 ;$ y Aceptación Neutral (NA) : $\alpha=.73$.

Las medias y desviaciones típicas obtenidas por los distintos grupos (altos y bajos en control de imagen y en viveza de imagen) en las puntuaciones de las distintas subescalas del Death Attitude Profile-Revised (DAPR): Aceptación de Acercamiento (AA), Miedo a la Muerte (FD), Evitación de la Muerte (DA), Aceptación de Escape (EA), y Aceptación Neutral (NA), se encuentran en la Tabla 1. Los resultados del MANOVA indicaron que no existían diferencias significativas entre los individuos altos y bajos en viveza de imagen mental (VVIQ) en las puntuaciones de las distintas subescalas del Death Attitude Profile-Revised (DAP-R): Aceptación de Acercamiento (AA), Miedo a la Muerte (FD), Evitación de la Muerte (DA), Aceptación de Escape (EA), y Aceptación Neutral (NA), Lambda del Wilks $=.98, F(5,272)=0.88, p=.50, \eta^{2}{ }_{p}=.02$, potencia $=.31$.

El control de imagen influyó significativamente en las distintas subescalas del Death Attitude ProfileRevised (DAP-R): Aceptación de Acercamiento (AA), Miedo a la Muerte (FD), Evitación de la Muerte (DA), Aceptación de Escape (EA), y Aceptación Neutral (NA), Lambda del Wilks $=.95, F(5,272)=2.92, p=.01$, $\eta^{2}{ }_{p}=.05$, potencia $=.85$. Los análisis univariados indicaron que existía diferencia significativa entre los estudiantes que tenían una alta capacidad de control de imagen (GTVIC) y los que tenían bajo control de imagen (GTVIC) en algunas de las subescalas del Death Attitude Profile-Revised (DAP-R): Miedo a la Muerte (FD), Evitación de la Muerte (DA), y Aceptación Neutral (NA). Los estudiantes que tenían un mayor control de imagen tuvieron significativamente menos Miedo a la Muerte (FD) $(M=3.81, S D=1.53)$ que los estudiantes que tuvieron un menor control de imagen $(M=4.19, S D=1.40), F(1,276)=4.12, p=.04, \eta^{2} p=.02$, potencia $=.53$. Los estudiantes que tenían un mayor control de imagen tuvieron menores puntuaciones en la escala de Evitación de la Muerte (DA) $(M=3.90, S D=1.71)$ que los estudiantes que tuvieron un menor control 
de imagen $(M=4.33, S D=1.39)$, y esta diferencia fue significativa, $F(1,276)=5.83, p=.02, \eta^{2} p=.02$, potencia $=.67$. Finalmente, los estudiantes que tenían un mayor control de imagen tuvieron significativamente mayores puntuaciones en la escala de Aceptación Neutral (NA) $(M=5.63, S D=1.12)$, que los estudiantes que tuvieron un menor control de imagen $(M=5.09, S D=1.25), F(1,276)=10.20, p=.002, \eta^{2} p=.04$, potencia $=.89$. El control de imagen no influyó significativamente en las demás subescalas del Death Attitude ProfileRevised (DAP-R): Aceptación de Acercamiento (AA), $F(1,276)=1.12, p=.66, \eta_{p}^{2}=.01$, potencia $=.07, \mathrm{y}$ Aceptación de Escape $(\mathrm{EA}), F(1,276)=0.02, p=.89, \eta^{2} p=.01$, potencia $=.05$. La interacción entre la viveza de imagen (VVIQ) y el control de imagen (GTVIC) no resultó significativa, Lambda del Wilks $=.98, F(5$, 272) $=1.31, p=.26, \eta_{p}^{2}=.02$, potencia $=.46$.

\section{Tabla 1}

Medias y Desviaciones Típicas (entre paréntesis) de las Escalas de Actitud ante la Muerte en Función de la Capacidad de Formar Imágenes Vivas (VVIQ), y del Control de Imagen (GTVIC) de Estudiantes de Educación Secundaria Obligatoria

\begin{tabular}{|c|c|c|c|c|c|}
\hline \multirow{2}{*}{$\begin{array}{l}\text { Death Attitude Profile- } \\
\text { Revised (DAP-R) }\end{array}$} & \multicolumn{2}{|c|}{$\begin{array}{l}\text { Vividness of Visual Imagery } \\
\text { Questionnaire (VVIQ) }\end{array}$} & \multicolumn{2}{|c|}{$\begin{array}{c}\text { Gordon Test of Visual Imagery } \\
\text { Control (GTVIC) }\end{array}$} & \multirow{2}{*}{$\begin{array}{c}\text { Total } \\
M(D T)\end{array}$} \\
\hline & Altos & Bajos & Altos & Bajos & \\
\hline Approach Acceptance (AA) & $3.61(1.20)$ & $3.67(1.28)$ & $3.62(1.33)$ & $3.67(1.13)$ & $3.64(1.25)$ \\
\hline Fear of Death (FD) & $4.04(1.49)$ & $3.93(1.48$ & $3.81(1.53)$ & $4.19(1.40)$ & $3.98(1.48)$ \\
\hline Death Avoidanc (DA) & $4.05(1.44)$ & $4.14(1.69)$ & $3.90(1.71)$ & $4.33(1.39)$ & $4.10(1.58)$ \\
\hline Escape Acceptance (EA) & $3.43(1.30)$ & $3.53(1.43)$ & $3.49(1.43)$ & $3.47(1.31)$ & $3.48(1.37)$ \\
\hline Neutral Acceptance (NA) & $5.20(1.15)$ & $5.54(1.23)$ & $5.63(1.12)$ & $5.09(1.25)$ & $5.39(1.21)$ \\
\hline
\end{tabular}

Nota: Altas puntuaciones en el VVIQ indican baja viveza de imagen, y viceversa

\section{Estudio 2}

El objetivo de esta investigación era saber si la viveza de imagen y el control de imagen influían en la actitud ante la muerte en un grupo de estudiantes universitarios. Para ello, formulamos la hipótesis de que, la viveza de imagen y el control de imagen que tienen los participantes influyen significativamente en su actitud ante la muerte. Las variables independientes son la viveza de imagen y el control de imagen de los participantes, y las variables dependientes son las subescalas del Death Attitude Profile-Revised (DAP-R): Aceptación de Acercamiento (AA), Miedo a la Muerte (FD), Evitación de la Muerte (DA), Aceptación de Escape (EA), y Aceptación Neutral (NA).

\section{Método}

\section{Participantes}

Un grupo de 250 individuos (49 hombres y 101 mujeres), estudiantes del tercer y cuarto curso de los grados del Campus de Ourense, de la Universidad de Vigo, con una media de edad de 22.03 años, una desviación típica de 2.38, y un rango de 19 a 28 años. La distribución por facultades fue la siguiente: Educación Primaria (49), Educación Infantil (39), Educación Social, (62), Trabajo Social (56), Ciencias Ambientales (18), Ciencia y Tecnología de los Alimentos (26).

\section{Instrumentos, Procedimiento y Análisis de Datos.}

Los instrumentos, el procedimiento y el análisis de datos fueron los mismos que el Estudios 1. La única diferencia entre el Estudio 1 y el Estudio 2 es que en el Estudio 1 se utilizaron estudiantes de Educación Secundaria Obligatoria, y en el Estudio 2 se utilizaron estudiantes universitarios.

\section{Resultados}

La fiabilidad del Gordon Test of Visual Imagery Control (TVIC; Richardson, 1969), medida a través del alfa de Cronbach fue .78, y la del Vividness of Visual Imagery Questionnaire (VVIQ) de .91. La fiabilidad de 
las distintas subescalas del Death Attitude Profile-Revised (DAP-R) fueron las siguientes: Aceptación de Acercamiento (AA) : $\alpha=.89$, Miedo a la Muerte (FD) $: \alpha=.85$, Evitación de la Muerte (DA) : $\alpha=.91$, Aceptación de Escape (EA) : $\alpha=.83$, y Aceptación Neutral (NA) : $\alpha=.72$.

Las medias y desviaciones típicas obtenidas por los distintos grupos (altos y bajos en control de imagen, y altos y bajos en viveza de imagen) en las puntuaciones de las distintas subescalas del Death Attitude ProfileRevised (DAP-R): Aceptación de Acercamiento (AA), Miedo a la Muerte (FD), Evitación de la Muerte (DA), Aceptación de Escape (EA), y Aceptación Neutral (NA), se encuentran en la Tabla 2. Los resultados del MANOVA indicaron que no existían diferencias significativas entre los individuos altos y bajos en imagen mental (VVIQ) en las puntuaciones de las distintas subescalas del Death Attitude Profile-Revised (DAP-R), Lambda de Wilks $=.98, F(5,240)=1.04, p=.39, \eta^{2} p=.02$, potencia $=.37$. El control de imagen tampoco influyó significativamente en las distintas subescalas del Death Attitude Profile-Revised (DAP-R): Aceptación de Acercamiento (AA), Miedo a la Muerte (FD), Evitación de la Muerte (DA), Aceptación de Escape (EA), y Aceptación Neutral (NA), Lambda de Wilks $=.99, F(5,240)=0.64, p=.67, \eta^{2} p=.01$, potencia $=.23$. La interacción entre la viveza de imagen (VVIQ) y el control de imagen (GTVIC) no resultó significativa, Lambda de Wilks $=.99, F(5,240)=0.72, p=.61, \eta_{p}=.02$, potencia $=.26$.

\section{Tabla 2}

Medias y Desviaciones Típicas (entre Paréntesis) de las Escalas de Actitud ante la Muerte, en Función de la Capacidad de Formar Imágenes Vivas (VVIQ), y del Control de Imagen (GTVIC) de Estudiantes Universitarios

\begin{tabular}{lccccc}
\hline & \multicolumn{2}{c}{$\begin{array}{c}\text { Vividness of Visual Imagery } \\
\text { Questionnaire (VVIQ) }\end{array}$} & \multicolumn{2}{c}{$\begin{array}{c}\text { Gordon Test of Visual Imagery } \\
\text { Control (GTVIC) }\end{array}$} & Total \\
\cline { 2 - 6 } Revised (DAP-R) & Altos & Bajos & Altos & Bajos & $M(D T)$ \\
\hline Approach Acceptance (AA) & $2.60(1.24)$ & $2.40(1.20)$ & $2.47(1.23)$ & $2.52(1.22)$ & $2.49(1.22)$ \\
Fear of Death (FD) & $4.32(1.34)$ & $3,93(1.50)$ & $3.99(1.45)$ & $4.28(1.41)$ & $4.11(1.44)$ \\
Death Avoidanc (DA) & $4.20(1.56)$ & $3.96(1.71)$ & $3.93(1.69)$ & $4.28(1.56)$ & $4.07(1.64)$ \\
Escape Acceptance (EA) & $3.13(1.46)$ & $3.02(1.49)$ & $3.09(1.55)$ & $3.04(1.37)$ & $3.07(1.48)$ \\
Neutral Acceptance (NA) & $5.30(0.97)$ & $5.52(0.82)$ & $5.51(0.87)$ & $5.29(0.93)$ & $5.42(0.90)$ \\
\hline
\end{tabular}

Nota: Altas puntuaciones en el VVIQ indican baja viveza de imagen, y viceversa

\section{Discusión}

El objetivo de esta investigación era saber si la viveza de imagen y el control de imagen influían en la actitud ante la muerte en un grupo de adolescentes (Estudio 1) y en un grupo de adultos (Estudio 2). Los coeficientes alfa de Cronbach del Vividness of Visual Imagery Questionnaire (VVIQ) fueron altas, tanto en el Estudio 1 (alfa $=.91)$, como en el Estudio 2 (alfa = .91). Esta puntuación es considerada por George y Mallery (2003) como "excelente", y es semejante a la encontrada en otros estudios que se realizaron con este test (Campos et al., 2002). El coeficiente alfa encontrado en el Gordon Test of Visual Imagery Control (TVIC; Richardson, 1969) fue de .68 en el Estudio 1, y de .78 en el Estudio 2. Las dos son “aceptables", según George y Mallery (2003). Este resultado es también semejante al utilizado en otras investigaciones con este test (Pérez-Fabello y Campos, 2004).

Los coeficientes alfa de Cronbach de las subescalas del Death Attitude Profile-Revised (DAP-R): Aceptación de Acercamiento (AA), Miedo a la Muerte (FD), Evitación de la Muerte (DA), Aceptación de Escape (EA), y Aceptación Neutral (NA) oscilaron, en el Estudio 1, entre .73 de la Aceptación Neutral (NA) que, según George y Mallery (2003) es "aceptable", y .87 de la subescala Aceptación de Acercamiento (AA), que según George y Mallery (2003) es "buena". En el Estudio 2, los coeficientes alfa oscilaron entre .72 de la subescala Aceptación Neutral (NA), que según George y Mallery (2003) es "aceptable", y .91 de la subescala Evitación de la Muerte (DA), que George y Mallery (2003) es "excelente". Estos resultados son semejantes a los obtenidos en otras investigaciones (López-Pérez et al. 2020a, b; Wong et al., 1994).

En el Estudio 1, hemos encontrado que el control de imagen influyó significativamente en algunas de las subescalas del Death Attitude Profile-Revised (DAP-R): Aceptación de Acercamiento (AA), Miedo a la 
Muerte (FD), Evitación de la Muerte (DA), Aceptación de Escape (EA), y Aceptación Neutral (NA). Los estudiantes de la Educación Secundaria Obligatoria que tenían un mayor control de imagen tuvieron significativamente menos puntuaciones en la escala de Miedo a la Muerte (FD) y en la escala de Evitación de la Muerte (DA) que los estudiantes que tuvieron un menor control de imagen. Parece que los alumnos que tienen un mayor control de sus imágenes controlan las imágenes de miedo a la muerte, y controlan también la evitación de la muerte, es decir, puntúan bajo en ítems como "evito totalmente pensar en la muerte", ya que son capaces de controlar la situación. Estos resultados coinciden con los obtenidos por López-Pérez et al. (2020a) en un grupo de estudiantes de la Educación Secundaria Obligatoria, y coincide también con los obtenidos por López-Pérez et al. (2020b) con estudiantes universitarios, en los que estudiaron la influencia de la imagen positiva y negativa de la muerte en las actitudes hacia la muerte. Es decir, parece que el control de imagen equivale a la imagen positiva de la muerte. Las personas que tienen un fuerte control de las imágenes mentales saben utilizar las imágenes positivas y controlar las negativas.

En nuestra investigación hemos encontrado también que los individuos altos en control de imagen (Estudio 1) tuvieron una puntuación más alta en la escala de Aceptación Neutral (NA) que los individuos con bajo control de imagen. Los altos en control de imagen ven la muerte "como un aspecto natural de la vida". LópezPérez et al. (2020a) en un grupo de adolescentes, y López-Pérez et al. (2020b) en un grupo de estudiantes universitarios también obtuvieron el mismo resultado en esta escala. Los estudiantes con imagen positiva de la muerte tuvieron una mayor puntuación en la escala de Neutral Acceptance (NA) que los estudiantes con una imagen negativa de la muerte.

En el Estudio 1 no hemos encontrado diferencias significativas entre los altos y bajos en control de imagen en las otras dos escalas del Death Attitude Profile-Revised (DAP-R): Aceptación de Acercamiento (AA) y Aceptación de Escape (EA), resultado que no coincide con los encontrados por López-Pérez et al. (2020a, b), que encontraron resultados positivos.

En el Estudio 2 no hemos encontrado diferencias significativas entre los estudiantes universitarios altos y bajos en control de imagen, en ninguna de las subescalas del Death Attitude Profile-Revised (DAP-R). Pensábamos encontrar, por lo menos, las mismas diferencias significativas que en el Estudio 1, pero parece que en los estudiantes universitarios, al contrario de lo que sucede en los estudiantes de la Educación Secundaria Obligatoria, el control de imagen no influye significativamente en ninguna de las actitudes antes la muerte. Este resultado plantea un gran número de interrogantes que deberán ser resueltos en sucesivas investigaciones.

En ambos estudios no hemos encontrado influencia de la viveza de imagen de los participantes, estudiantes de la Educación Secundaria Obligatoria y estudiantes universitarios, en ninguna de las subescalas del Death Attitude Profile-Revised (DAP-R). Como hemos visto en la introducción, muchos estudios relacionaban la imagen de la muerte con algunas actitudes hacia la muerte (López-Pérez et al., 2020a, b), y con muchos desórdenes mentales (Berna et al. 2011; Boelen y Huntjens, 2008; Brewin et al. 2010; Deeprose y Holmes, 2010; Hales et al. 2011; Holmes et al., 2008). A pesar de que estos comportamientos están directamente relacionados con las imágenes mentales, parece que la capacidad de los individuos de formar imágenes vivas no se relaciona con ninguna de las actitudes hacia la muerte. Este resultado también presenta muchos interrogantes que deberán ser resueltos en futuras investigaciones.

Este trabajo tiene la limitación de que sólo se utilizan dos tipos de imagen, la viveza de imagen y el control de imagen, y dos tipos concretos de edad. Sería interesante utilizar otros tipos de imagen y otras edades para analizar si la viveza de imagen y el control de imagen influyen en las actitudes hacia la muerte. En esta relación entre la viveza de imagen y el control de imagen con las actitudes ante la muerte, también sería interesante analizar la influencia del género y la cultura. Como los datos se han recogido antes del COVID-19, también sería interesante recoger los mismos datos después de la pandemia para analizar la relación del control de imagen y la viveza de imagen con las actitudes ante la muerte. Pensamos que el miedo que hemos sentido todos ante la pandemia, y la cantidad de imágenes que hemos visto, pueden influir en la relación entre la imagen mental y la actitud ante la muerte. 
Nuestros resultados pueden tener una amplia repercusión en el ámbito escolar. El alumnado debe tener una educación emocional, y entre los temas a tratar en la educación emocional es el miedo y la ansiedad ante la muerte. Una buena estrategia educativa puede ser controlar las imágenes negativas de la muerte y convertirlas en imágenes positivas, y de este modo, reducir el miedo y la ansiedad.

\section{Referencias}

Berna, C., Vincent, K., Moore, J., Tracey, I., Goodwin, G. M., \& Holmes, E. A. (2011). Presence of mental imagery associated with chronic pelvic pain: A pilot study. Pain Medicine, 12 (7), 1086-1093. https://doi.org/10.1111/j.1526-4637.2011.01152.x

Boelen, P. A., \& Huntjens, R. J. C. (2008). Intrusive images in grief: An exploratory study. Clinical Psychology and Psychotherapy, 15, 2017-226. https://doi.org/10.1002/cpp.568

Borkovec, R. D., Alacine, O, \& Behar, E. (2004). Avoidance theory of worry and generalized anxiety disorder. En R. G. Heimberg, C. L. Turk, \& D. S Mennin (Eds.). Generalized anxiety disorder: Advances in research and practice (pp. 77-108). The Guilford Press.

Brewin, C. R., Gregory, J. D., Lipton, M., \& Burgess, N. (2010). Intrusive images in psychological disorders: Characteristics, neural mechanisms, and treatment implications. Psychological Review, 117, 210-232. https://doi.org/10.1037/a0018113

Campos, A. (Ed.) (1998). Imágenes mentales. Servicio de Publicaciones de la Universidad de Santiago de Compostela.

Campos, A., \& Campos-Juanatey, D. (2019). Do gender, discipline and mental rotation influence orientation on "You-Are-Here-Maps". SAGE Open, 1-7. https://doi.org/10.1177/2158244019898800

Campos, A. \& Canto, M. (2019). Diferencias en imagen mental entre individuos españoles e italianos. Revista de Estudios e Investigación en Psicología y Educación, 6(1), 37-43. https://doi.org/10.17979/reipe.2019. 6.1.5271

Campos, A., \& Fuentes, L. (2016). Musical studies and the vividness and clarity of auditory imagery. Imagination, Cognition and Personality, 36(1), 75-84. https://doi.org/10.1177/0276236616635985

Campos, A., \& González, M. A. (2017). Importancia de las imágenes mentales en el pensamiento. Revista Mexicana de Investigación en Psicología, 9(2), 113-119. https://www.revistamexicanadeinvestigacion enpsicologia.com/index.php/RMIP/article/view/265

Campos, A., González, M. A., \& Amor, A. (2002). The Spanish version of the Vividness of Visual Imagery Questionnaire: Factor structure and internal consistency reliability. Psychological Reports, 90, 503-506. https://doi.org/10.2466/PR0.90.2.503-506

Campos, A., \& Pérez-Fabello, M. J. (2005). The Spanish version of Betts`Questionnaire upon Mental Imagery. Psychological Reports, 96(1), 51-56. https://doi.org/10.2466/pr0.96.1.51-56

Clark, D. M., Ehlers, A., Hackmann, A., McManus, F., Fennell, M., Grey, N., Waddington, L., \& Wild, J. (2006). Cognitive therapy versus exposure and applied relaxation in social phobia: A randomized controlled trial. Journal of Consulting and Clinical Psychology, 74(3), 568-578. https://doi.org/10.1037/0022006x.74.3.568

Deeprose, C., \& Holmes, E. (2010). An exploration of prospective imagery: The Impact of Future Events Scale. Behavioural and Cognitive Psychotherapy, 38(2), 201-209. https://doi.org/10.10.17/S135246580999671

Dönmez, C. F., \& Bilgin, J. (2020). The effect of guided imagery on death anxiety, empathy and attidude towards terminally ill patients in oncology nurses. Journal of Nursing \& Healtcare, 5(1), 1-10. https://doi.org/10.33140/JNH.05.01.04

George, D., \& Mallery, P. (2003). SPSS for Windows step by step: A simple guide and reference.11.0 update. Allyn \& Bacon.

Gesser, G., Wong, P., \& Reker, G. (1988). Death attitudes across the life-span: The development and validation of the Death Attitude Profile. Omega - Jounal of Death and Dying, 18, 113-128. https://doi.org/10.2190/ ODQB-7Q1E-2BER-H6YC 
Hales, S. A., Deeprose, C., Goodwin, G.M., \& Holmes, E. A. (2011). Cognitions in bipolar affective disorder and unipolar depression: Imagining suicide. Bipolar Disorders, 13(7-8), 651-661. https://doi.org/10.1111/ j.1399-5618.2011.00954.x

Holmes, E. A., Geddes, J. R., Colom, F., \& Goodwin, G. M. (2008). Mental imagery as an emotional amplifier: Application to bipolar disorder. Behaviour Research and Therapy, 46(12), 1251-1258. https://doi.org/ 10.1016/j.brat.2008.09.005

Holmes, E. A., \& Matheus, A. (2005). Mental imagery and emotion: A special relationship? Emotion, 5(4), 489-497. https://doi.org/10.1037/1528-3542.5.4.489

Lee, S. K. (2009). East Asian attitudes toward death - a search for the ways to help east Asian elderly dying in contemporary America. Permanente Journal, 13 (3), 55-60. https://doi.org/10.7812/TPP/08-068

López-Pérez, M. E., Pino-Juste, M., \& Campos, A. (2020a). Mental imagery and anxiety towards death in adolescence. (Manuscrito enviado para publicación).

López-Pérez, M. E., Pino-Juste, M., \& Campos, A. (2020b). Image of death and attitude towards death. North American Journal of Psychology, 22(4), 701-710.

Marks, D. F. (1973). Visual imagery differences in the recall of pictures. British Journal of Psychology, 64, 17-24. https://doi.org/10.1111/j.2044-8295.1973.tb01322.x

McGovern, M., \& Barry, M. M. (2000). Death education: Knowledge, attitudes, and perspectives of Irish parents and teachers. Death Studies, 24, 325-333. https://doi.org/10.1080/074811800200487

Ng, A. S., Abbott, M. J., \& Hunt, C. (2014). The effect of self-imagery on symptoms and process in social anxiety: A systematic review. Clinical Psychology Review, 34, 620-633. https://doi.org/10.1016/j.cpr. 2014.09.003

Nilsson, J., Lundh, L., \& Viborg, G. (2012). Imagery rescripting of early memories in social anxiety disorder: An experimental study. Behaviour Research and Therapy, 50, 387-392. https://doi.org/10.1016/j-brat.2012.03.004

Pérez-Fabello, M. J., \& Campos, A. (2004). Factor structure and internal consistency of the Spanish version of the Gordon Test of Visual Imagery Control. Psychological Reports, 94, 761-766. https://doi.org/10.2466/pr0.94.3.761-766

Pérez-Fabello, M. J., \& Campos, A. (2017). Dissociative experiences and vividness of auditory imagery. Creativity Research Journal, 29(2), 200-205. https://doi.org/10.1080/10400419.2017.1303310

Pérez-Fabello, M. J., Campos, A., \& Felisberti, F. (2018). Object-spatial imagery in Fine Arts, Psychology and Engineering. Thinking Skills and Creativity, 27, 131-138. https://doi.org/10.1016/j.tsc.2017.12.005

Richardson, A. (1969). Mental imagery. Routledge \& Kagan Paul.

Stopa, L., Brown, M. a., \& Hirsch, C. R. (2012). The effects of repeated imagery practice on self-concept, anxiety, and performance in socially anxious participants. Journal of Experimental Psychopathology, 3(2), 223-242. https://doi.org/10.5127/jep.021511

WeBlau, C., Closs, M., Höfling, V., \& Steil, R. (2015). Visual mental imagery and symptoms of depression - results from a large-scale web-based study. BMC Psychiatry, 15, 308. https://doi.org/10.1186/s12888-015-0689-1

Wild, J., Hackmann, A., \& Clark, D. M. (2008). Rescripting early memories linked to negative images in social anxierty disorder: A pilot study. Behavior Therapy, 39(1), 47-56. https://doi.org/10.1016/j.beth. 2007.04.003

Wong, P., Reker, G., \& Gesser, G. (1994). Death Attitude Profile-Revised: A multidimensional measure of attitudes toward death. En R. A. Neimeyer (Ed.). Death anxiety handbook: Research, instrumentation, and application (pp. 121-148). Taylor and Francis.

Fecha de recepción: 16 de septiembre de 2020.

Fecha de revisión: 27 de octubre de 2020.

Fecha de aceptación: 29 de octubre de 2020.

Fecha de publicación: 1 de diciembre de 2020. 